\title{
Transient velocity overshoot dynamics in GaAs for electric fields $\leqslant 200 \mathrm{kV} / \mathrm{cm}$
}

\author{
J. Son, W. Sha, J. Kim, T. B. Norris, J. F. Whitaker, and G. A. Mourou \\ Center for Ultrafast Optical Science, Department of Electrical Engineering and Computer Science, \\ University of Michigan, 2200 Bonisteel Blvd., 1006 IST, Ann Arbor, Michigan 48109-2099
}

(Received 5 April 1993; accepted for publication 17 June 1993)

\begin{abstract}
We have experimentally studied the transient velocity overshoot dynamics of photoexcited carriers in GaAs for electric fields as great as $200 \mathrm{kV} / \mathrm{cm}$. Time domain waveforms proportional to the velocity and the acceleration of carriers have been acquired, respectively, from guided and free-space radiating signals which contain terahertz frequency components. The measurements demonstrated that the degree of overshoot was maximized for an electric field on the GaAs between 40 and $50 \mathrm{kV} / \mathrm{cm}$ when 1.44-eV photons in an $80-$ fs laser pulse excited the sample. For carriers excited with higher initial energy $(1.55 \mathrm{eV})$, the degree of overshoot decreased and the maximum degree of overshoot occurred at a higher electric field.
\end{abstract}

Hot-electron effects which lead to such ultrafast phenomena as ballistic carrier transport and velocity overshoot have been utilized in recent years in the conceptualization of numerous high-speed semiconductor devices. ${ }^{1}$ The dynamics of carriers in electric fields of up to several hundred kilovolts per centimeter in such devices can be described by an initial ballistic, high-velocity regime, followed by a velocity overshoot resulting from intervalley scattering of electrons. Therefore, substantive physical information on the behavior of velocity overshoot is of great importance in the design of ultrasmall, high-speed transistors. Nearly all the studies of time-domain velocity overshoot phenomena, however, have concentrated only on theoretical approaches such as Monte Carlo simulations. ${ }^{2}$ The direct experimental investigation of transport dynamics has been considerably enhanced by the rapid development of stable, tunable near-infrared femtosecond lasers ${ }^{3}$ and high temporal-resolution measurement techniques.

The study of time-domain velocity overshoot using photogenerated and guided transients, which were measured using the electro-optic (EO) sampling technique, was first undertaken by Meyer et al. ${ }^{4}$ This work, however, was rather qualitative due to limitations of the femtosecond laser source and the device structure studied. In this investigation, we have optically excited $\mathrm{GaAs}$ to produce free-space radiating terahertz $(\mathrm{THz})$ waveforms, which serve as a probe of the temporal dynamics of the carriers in the semiconductor. ${ }^{5,6}$ In addition, we have also reproduced the experiment of Meyer et al., with two significant improvements: we used laser wavelengths tuned close to the band gap of GaAs, and a device which exhibited a spatially uniform electric field up to much higher bias values. By proper sample design and control of the optical pump intensity and wavelength, we have been able, for the first time, to study velocity overshoot dynamics under constant electric field conditions up to $200 \mathrm{kV} / \mathrm{cm}$.

A $p-i-n$ structure embedded in a coplanar stripline was utilized in both the $\mathrm{THz}$ radiation and the guided-transient experiments. Carriers were photogenerated in the $i$ region of the devices and their time-dependent velocity determined. This was possible since the transient velocity creates a time-dependent photocurrent in the $i$ region, produc- ing a guided signal on the transmission line which is measured using the EO sampling technique. ${ }^{7}$ In addition, the transient acceleration of the carriers produced a radiating free-space signal which was detected by a standard THz photoconductive sampling technique. ${ }^{8}$ The two measurements were performed on the same sample and were complementary, allowing a direct comparison of the carrier velocity from the guided-wave experiment with the integral of the carrier acceleration from the radiated-wave experiment. The electric-field and initial-carrier-energy dependencies of velocity overshoot were successfully investigated in the subpicosecond time scale using these techniques.

The photoconductive sampling technique utilized in the detection of the THz signals has a very high signal-tonoise ratio, allowing the collection of weak signals from the $p-i-n$ transmitter. Direct information on the acceleration of carriers is obtained when the following conditions are achieved: the detection should be done in the far field, the detector gate should be short compared to the time scale of the dynamic carrier response, the carrier density should be constant after the optical injection, and the electric field should maintain the same strength after the radiation due to the carrier acceleration. These conditions were reasonably met in our experiments.

The reverse-biased lateral $p-i-n$ structure was used in order to provide a uniform electric field in the undoped GaAs region of the device. The ohmic contacts were made by the deposition of $p$ and $n$ metals, $\mathrm{Au} / \mathrm{Zn} / \mathrm{Ni} / \mathrm{Au}$, and $\mathrm{Ni} / \mathrm{Ge} / \mathrm{Au} / \mathrm{Ti} / \mathrm{Au}$, respectively, which were annealed after each deposition (see Fig. 1). The separation between the $p$ and $n$ contacts, the $i$ region, was $4-\mu \mathrm{m}$ wide, and the electrode width was $50 \mu \mathrm{m}$. Breakdown occurred at the nominal electric field of $205 \mathrm{kV} / \mathrm{cm}$ ( $82 \mathrm{~V}$ across $4 \mu \mathrm{m}$ ). The $i$ region was photoexcited with $80-\mathrm{fs}$ optical pulses from a self-mode-locked Ti:sapphire laser, ${ }^{3}$ which was tuned between 800 and $860 \mathrm{~nm}$ to excite carriers in the $\Gamma$ valley with different initial energies. Also, the average power of the pump beam was kept below $0.5 \mathrm{~mW}$, to avoid generating so many carriers that the applied field would be screened, as discussed below.

For the $\mathrm{THz}$ radiation measurement, the lateral $p-i-n$ 

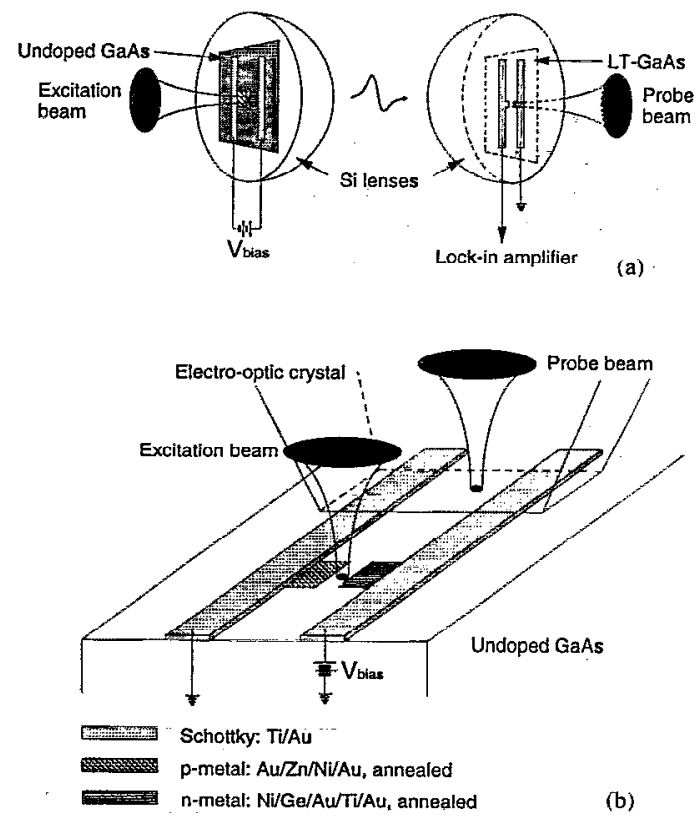

FIG. 1. Experimental configuration for observation of velocity overshoot: (a) terahertz radiation technique and (b) guided photocurrent measurement using electro-optic sampling.

diode, fabricated on a $500-\mu \mathrm{m}$-thick GaAs layer, was clamped to a $12.7-\mathrm{mm}$-diam hyperhemispherical Si lens to collimate the radiation resulting from the optical excitation. This is illustrated in Fig. 1(a). The radiated signal was detected by a photoconductive-receiver antenna, which was fabricated on low-temperature-grown GaAs and had a gate duration of $<0.5$ ps full width at half maximum (FWHM). The detector was also mounted on a Si lens.

For the EO sampling measurement of the transient velocity, the $p-i-n$ structure was embedded in a coplanar stripline as shown in Fig. 1(b). The guided signal was measured using an external electro-optic sampling crystal of $\mathrm{LiTaO}_{3}$, as also shown in the figure. The waveforms were measured after only $50 \mu \mathrm{m}$ of propagation from the $p-i-n$ structure, in order to limit the dispersion and attenuation effects of the transmission line.

Both the $\mathrm{THz}$ radiation and the photocurrent on the transmission line were measured over a wide range of electric fields (E), up to $200 \mathrm{kV} / \mathrm{cm}$. The amplitude of the positive peak of the THz waveform (see Fig. 2) increased as $\mathbf{E}$ increased, reaching a maximum at $90 \mathrm{kV} / \mathrm{cm}$, and then slightly decreased as $\mathbf{E}$ was raised further. The peak of the waveform at $190 \mathrm{kV} / \mathrm{cm}$ bias was nearly $20 \%$ lower than the maximum peak at $90 \mathrm{kV} / \mathrm{cm}$. This peak and the subsequent rolloff in the $\mathrm{THz}$ pulse amplitude for $\mathbf{E}>90$ $\mathrm{kV} / \mathrm{cm}$ indicated that at very high $\mathbf{E}$, the maximum electron acceleration saturated. In addition, the positive peak (i.e., the position of the maximum acceleration) appeared at earlier times as $\mathbf{E}$ was increased, as noted by the $300-\mathrm{fs}$ difference in the arrival time of the peaks between 0.5 and $190 \mathrm{kV} / \mathrm{cm}$ bias conditions. This was expected as was the very similar behavior of the peak of the guided photocurrent measured by the EO sampling technique.

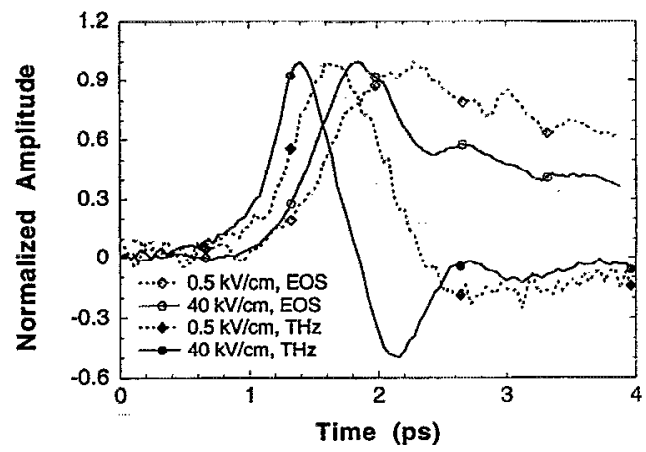

FIG. 2. Time-domain waveforms of transient photocurrent signals guided on a transmission line (measured via electro-optic sampling, EOS) and radiated from a broadband antenna ( $\mathrm{THz}$ radiation), vs external dc-bias electric fields. The excitation wavelength was $860 \mathrm{~nm}(1.44 \mathrm{eV})$.

While the positive portion of the $\mathrm{THz}$ radiation signal represented the carrier acceleration, the negative part represented the deceleration. In Fig. 2, the $\mathrm{THz}$ waveform at $0.5 \mathrm{kV} / \mathrm{cm}$ is observed to be essentially unipolar, while at $40 \mathrm{kV} / \mathrm{cm}$ it is bipolar, indicating an overshoot in the photocurrent at higher fields (for an excitation wavelength of $860 \mathrm{~nm}$ ). This was also evident in the EO sampling waveform shown in the same figure. It was evident from these data that the $\mathrm{THz}$ waveforms appeared very similar to the first derivative of the EO sampling waveforms. Therefore, in Fig. 3, the THz-radiation waveform at $40 \mathrm{kV} / \mathrm{cm}$ was integrated and compared with the EO sampling waveform at the same field. While the agreement is outstanding, a diminished peak in the integrated waveform of the $\mathrm{THz}$ signal is apparent, arising from the slower experimental response in the photoconductive relative to the EO sampling measurements.

As mentioned earlier, it is possible for screening of the applied electric field, due to the photoexcited carriers, to lead to this type of time-domain overshoot. To prove that the electric field in the gap remained constant during the photocurrent overshoot, we performed an experiment in which two equal-intensity pump beams separated in time by $10 \mathrm{ps}$ were incident on the gap. We found that the amplitude of the photocurrent due to the second pulse, when both the beams illuminated the sample, was still $95 \%$

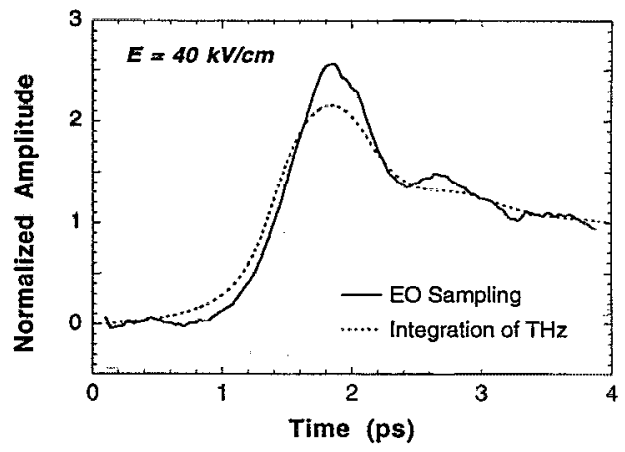

FIG. 3. Comparison between the integration of the THz-radiation waveform and the direct EO-sampling measurements of the guided waveform. The signals were normalized with respect to their steady-state values. 


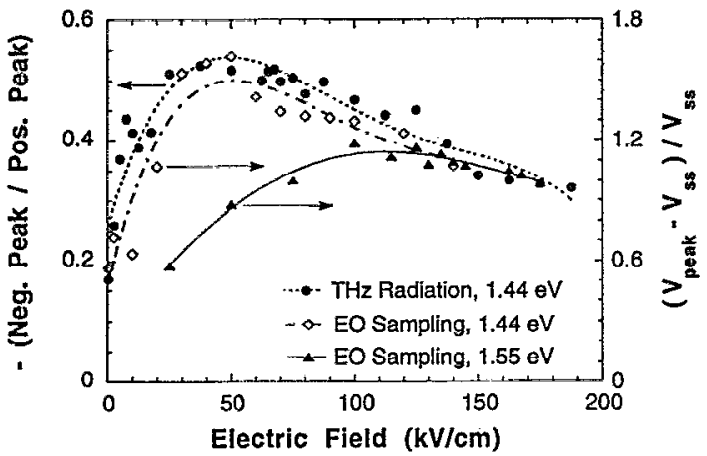

FIG. 4. The degree of overshoot with respect to the applied electric field. The maxima in this figure of merit occur at $40-50 \mathrm{kV} / \mathrm{cm}$ for both guided (EO) and radiated (THz) transient photocurrent experiments with an excitation of $1.44 \mathrm{eV}(860 \mathrm{~nm})$. For carriers with the higher initial energy of $1.55 \mathrm{eV}(800 \mathrm{~nm})$, the degree of overshoot is smaller, and the maximum degree of overshoot occurs at a higher electric field. The lines in this figure are only a guide to the eye.

of the amplitude produced when the first pulse was blocked. Thus, field screening appears to perturb our results only slightly, so that we can attribute the bipolar waveform of the $\mathrm{THz}$ experiment and the overshoot waveform of the guided photocurrent experiment to velocity overshoot due to the $k$-space transfer of electrons from the $\Gamma$ to $L$ or $X$ valleys, and not to dynamic field collapse. ${ }^{6}$

We have identified quantities which describe the degree of velocity overshoot and plotted their dependence on the bias electric field in Fig. 4. For the $\mathrm{THz}$ radiation experiment, the degree of overshoot was defined as the ratio of the magnitudes of the negative and positive peaks, while for the guided photocurrent experiment, it was defined as the difference of the peak amplitude and the steady-state amplitude divided by the latter. Although slightly different definitions were applied to these two experimental techniques, both showed the maximum overshoot effect around $40-50 \mathrm{kV} / \mathrm{cm}$ in the case of $860-\mathrm{nm}$ $(1.44 \mathrm{eV})$ laser excitation.

With the tuning of laser wavelength from 860 to 800 $\mathrm{nm}(1.55 \mathrm{eV})$, we have observed the decrease of overshoot and the shift of maximum overshoot to higher bias fields as shown in Fig. 4. This result actually has been predicted in
Monte Carlo simulations by Wysin et al. ${ }^{9}$ As the carriers are excited higher into the $\Gamma$ valley of the conduction band, the time for carriers to scatter into the upper valleys is reduced, resulting in a decreased peak average velocity.

The diminishing overshoot phenomenon at very high fields at all initial carrier energies might be explained by the carriers in satellite valleys maintaining high steadystate velocity at very high electric fields. In conclusion, for the first time, transient carrier transport dynamics in GaAs at very high fields (up to $200 \mathrm{kV} / \mathrm{cm}$ ) have been investigated experimentally. We have measured the high-field velocity and acceleration of carriers using two separate experimental techniques on the same device, allowing a detailed confirmation of the observation of velocity overshoot. The comparison has shown that the THz radiation due to a rapidly time-varying photocurrent is the time derivative of this same photocurrent guided on a transmission line and measured by EO sampling. These measurements were also analyzed quantitatively to give the electric field and initial-carrier-energy dependencies of transient velocity overshoot in GaAs.

The authors thank June-Koo Rhee for useful discussions and technical assistance. This research was supported by AFOSR contract number AFOSR-90-0214 (University Research Initiative) and by the National Science Foundation through Center for Ultrafast Optical Science under STC PHY 8920108.

${ }^{1}$ See, for general review, K. Hess and G. J. Iafrate, Proc. IEEE 76, 518 (1988).

${ }^{2}$ E. Constant, in Hot-Electron Transport, edited by L. Reggiani (Springer, Berlin, 1988), p. 227.

${ }^{3}$ D. E. Spence, P. N. Kean, and W. Sibbet; Opt. Lett. 16, 42 (1991).

${ }^{4}$ K. Meyer, M. Pessot, G. Mourou, R. Grodin, and S. Chamoun. Appl. Phys. Lett. 53, 2254 (1988).

${ }^{5}$ L. Xu, X.-C. Zhang, and D. H. Auston, Appl. Phys. Lett. 59, 3357 (1991).

${ }^{6}$ W. Sha, J. Rhee, T. B. Norris, and W. J. Schaff, IEEE J. Quantum Electron. 28, 2445 (1992).

${ }^{7}$ J. A. Valdmanis and G. A. Mourou, IEEE J. Quantum Electron. 22, 69 (1986).

${ }^{8}$ M. van Exter and D. R. Grischkowsky, IEEE Trans. Microwave Theory Tech. 38, 1684 (1990).

${ }^{9}$ G. M. Wysin, D. L. Smith, and A. Redondo; Phys. Rev. B 38, 12514 (1988). 